\title{
Influence of asymmetry and nodal planes on high-harmonic generation in heteronuclear molecules
}

\author{
B. B. Augstein and C. Figueira de Morisson Faria \\ Department of Physics and Astronomy, University College London, Gower \\ Street, London WC1E 6BT, United Kingdom
}

\begin{abstract}
The relation between high-harmonic spectra and the geometry of the molecular orbitals in position and momentum space is investigated. In particular we choose two isoelectronic pairs of homonuclear and heteronuclear molecules, such that the highest occupied molecular orbital of the former exhibit at least one nodal plane. The imprint of such planes is a strong suppression in the harmonic spectra, for particular alignment angles. We are able to identify two distinct types of nodal planes. If the nodal planes are determined by the atomic wavefunctions only, the angle for which the yield is suppressed will remain the same for both types of molecules. In contrast, if they are determined by the linear combination of atomic orbitals at different centers in the molecule, there will be a shift in the angle at which the suppression occurs for the heteronuclear molecules, with regard to their homonuclear counterpart. This shows that, in principle, molecular imaging, which uses the homonuclear molecule as a reference and enables one to observe the wavefunction distortions in its heteronuclear counterpart, is possible.
\end{abstract}

PACS numbers: 33.80.Rv, 42.65.Ky 
Influence of asymmetry and nodal planes on high-harmonic generation in heteronuclear molecules 2

\section{Introduction}

High harmonic generation, first discovered in the late 1980s [1, is a process which occurs when atoms or molecules are exposed to intense laser fields, and results in the emission of high frequency coherent radiation. It can be readily understood in terms of the three step model, 2, 3, where an electron is ionized from the atom or molecule, propagates in the laser field, and recombines with its parent ion, upon which high harmonics are released.

Currently, there is much interest in this process due to the possibility of tomographic orbital reconstruction [4, 5, 6], and quantum interference effects [7, 8, 9. In particular quantum-interference minima and maxima due to high-harmonic emission at spatially separated centers, and high-harmonic suppression due to the presence of nodal planes in the orbital wavefunctions, have been identified and discussed theoretically. Such studies have been extensively performed in diatomic molecules 6 , 7, 8, 9, 10, 13, 17. In many of these studies so far, homonuclear molecules with definite orbital symmetry, either gerade or ungerade, have mostly been used, whereas heteronuclear molecules driven by strong fields are now starting to attract attention [11. The orbital wavefunctions in such molecules do not have a definite orbital symmetry, and heteronuculear molecules contain a static dipole moment. These attributes will affect the harmonic spectra and other strong field phenomena. Hence, a legitimate question to ask is whether two-center interference patterns in the spectra, or the imprint of nodal planes in form of high-harmonic suppression, can still be observed in the spectra.

In this work, we compare isoelectronic homonuclear and heteronuclear molecules, with particular emphasis on nodal planes in the position space wavefunction and twocenter interference effects. We assume the Born-Oppenheimer approximation to be valid, which should hold for large enough molecules 9 . Specifically, we take the pairs $\mathrm{Be}_{2}$ and $\mathrm{LiB}$, and $\mathrm{O}_{2}$ and NF. The homonuclear molecule in former and latter pair have a $\sigma_{u}$ and $\pi_{g}$ highest occupied molecular orbital (HOMO), respectively. Such orbitals contain nodal planes which are altered when a heteronuclear counterpart is chosen. In our investigations, we employ the single-active electron and the strong-field approximation and assume that the HOMO is the only active orbital. The influence of multiple orbitals has been discussed elsewhere [6, 13, 14.

This work is organized as follows. In Sec. 2 the three step model (Sec. 2.1), molecular orbital construction (Sec. 2.2) and interference conditions (Sec. 2.3) are discussed. In Sec. 3, we present our results. This section is split into two main parts. First, in Sec. 3.1, we address the molecular orbitals obtained by us, with a particular emphasis on their nodal planes, or the absence thereof for heteronuclear molecules. Subsequently, in Sec. 3.2 we discuss how such characteristics manifest themselves in the spectra. Finally, in Sec 4 we state the main conclusions to be drawn from this work.

\section{Model}

\subsection{Saddle-point equations}

The transition amplitude for high harmonic generation, within the framework of of the strong field approximation (SFA) [2], reads as, 
Influence of asymmetry and nodal planes on high-harmonic generation in heteronuclear molecules3

$$
\begin{aligned}
b_{\Omega}= & -i \int_{-\infty}^{\infty} d t \int_{-\infty}^{t} d t^{\prime} \int d^{3} k a_{\mathrm{rec}}^{*}(\mathbf{k}+\mathbf{A}(t)) a_{\mathrm{ion}}\left(\mathbf{k}+\mathbf{A}\left(t^{\prime}\right)\right) \\
& \exp \left[i S\left(t, t^{\prime}, \Omega, \mathbf{k}\right)\right]+\text { c.c. },
\end{aligned}
$$

with the action

$$
S\left(t, t^{\prime}, \Omega, \mathbf{k}\right)=-\frac{1}{2} \int_{t^{\prime}}^{t}[\mathbf{k}+\mathbf{A}(\tau)]^{2} d \tau-E_{0}\left(t-t^{\prime}\right)+\Omega t
$$

where $a_{\text {rec }}(\mathbf{k}+\mathbf{A}(t))=\left\langle\mathbf{k}+\mathbf{A}(t)\left|\mathbf{d} \cdot \mathbf{e}_{x}\right| \Psi\right\rangle$ and $a_{\text {ion }}\left(\mathbf{k}+\mathbf{A}\left(t^{\prime}\right)\right)=$ $\left\langle\mathbf{k}+\mathbf{A}\left(t^{\prime}\right)\left|\mathbf{E}\left(t^{\prime}\right) \cdot \mathbf{r}\right| \Psi\right\rangle$, are the recombination and ionization prefactors respectively. The terms $\mathbf{d}, \mathbf{e}_{x}$ and $\Omega$ denote the dipole operator and the laser-polarization vector and the harmonic frequency, respectively. The ionization potential of the highest occupied molecular orbital, denoted by $|\Psi\rangle$, is given by $E_{0}$. The length gauge is used throughout as the interference minima in the harmonic spectra vanish in the velocity gauge [15, and the length form of the dipole operator is used as it accounts for the presence of the static dipole moment $\mathrm{t}$.

To solve Eq. (10) we use the saddle-point approximation [16, where the action is stationary, such that $\partial S\left(t, t^{\prime}, \Omega, \mathbf{k}\right) / \partial t^{\prime}=\partial S\left(t, t^{\prime}, \Omega, \mathbf{k}\right) / \partial t=0$ and $\partial S\left(t, t^{\prime}, \Omega, \mathbf{k}\right) / \partial \mathbf{k}=\mathbf{0}$. This results in the saddle-point equations

$$
\begin{gathered}
\frac{\left[\mathbf{k}+\mathbf{A}\left(t^{\prime}\right)\right]^{2}}{2}+E_{0}=0, \\
\int_{t^{\prime}}^{t} d \tau[\mathbf{k}+\mathbf{A}(\tau)]=\mathbf{0} .
\end{gathered}
$$

and

$$
\frac{[\mathbf{k}+\mathbf{A}(t)]^{2}}{2}+E_{0}=\Omega
$$

These equations have the following physical interpretation. Initially the electron is tunnel ionized from the binding potential. This results in Eq. (3), which gives the conservation of energy. Note that this equation has only complex solutions, as a consequence of the fact that tunneling has no classical counterpart. Secondly, Eq. (4) results in the electron momentum in the continuum being constrained such that the electron returns to its place of origin, which we take to be the geometric center of the molecule. Finally, Eq. (5) represents conservation of energy at recombination.

\subsection{Molecular orbitals and dipole matrix elements}

The molecular orbitals can be calculated using the Linear Combination of Atomic Orbitals (LCAO) approximation, along with the Born Oppenheimer approximation. Under these assumptions, the molecular orbital wavefunction is given by

$$
\begin{aligned}
\Psi(\mathbf{r}) & =\sum_{\alpha} c_{\alpha}^{(L)} \Phi_{\alpha}^{(L)}(\mathbf{r}+\mathbf{R} / 2) \\
& +(-1)^{l_{\alpha}-m_{\alpha}+\lambda_{\alpha}} c_{\alpha}^{(R)} \Phi_{\alpha}^{(R)}(\mathbf{r}-\mathbf{R} / 2)
\end{aligned}
$$

$\ddagger$ Recently, we have shown that if a Gaussian basis set is employed in the modeling of the molecular orbitals, the velocity form of the dipole operator leads to a vanishing static dipole moment in heteronuclear molecule. This problem is absent if the length form is taken. For details see [17]. 
where $\mathbf{R}$ is the internuclear separation, $l_{\alpha}$ is the orbital quantum number and $m_{\alpha}$ is the magnetic quantum number. We assume that the ions are positioned along the $\mathrm{z}$ axis, i.e., that $\mathbf{R}=R \hat{e}_{z}$. The coefficients $c_{\alpha}^{(\xi)}$ form the linear combination of atomic orbitals and are extracted from the quantum chemistry code GAMESSUK [12. The indices $(L)$ and $(R)$ refer to the left or to the right ion, respectively, which is a distinction required for heteronuclear molecules, whereas for homonuclear molecules, $c_{\alpha}^{(L)}=c_{\alpha}^{(R)}=c_{\alpha}$ and $\Phi_{\alpha}^{(R)}=\Phi_{\alpha}^{(L)}=\Phi_{\alpha}$. The internuclear axis is taken to be along the $z$ direction, and the laser-field polarization is chosen along the radial coordinate. The parameter $\lambda_{\alpha}$ determines the orbital symmetry of the molecular orbital, with $\lambda_{\alpha}=\left|m_{\alpha}\right|$ and $\lambda_{\alpha}=\left|m_{\alpha}\right|+1$ for homonuclear molecules of gerade and ungerade symmetry, respectively.

The wavefunctions themselves are then expanded as Gaussian type orbitals with a real 6-31 basis set, with

$$
\Phi_{\alpha}^{(\xi)}(\mathbf{r})=\sum_{\nu} b_{\nu}^{(\xi)}\left(r_{\chi}\right)^{l_{\alpha}} e^{-\zeta_{\nu}^{(\xi)} r^{2}}
$$

We find that polarized basis sets make little difference to the orbital wavefunctions. For the $\sigma, \pi_{x}$ and $\pi_{y}$ orbitals, $r_{\chi}=z, x$ and $y$, respectively. The contraction coefficients, $b_{\nu}^{(\xi)}$ and exponents, $\zeta_{\nu}^{(\xi)}$, are also obtained from GAMESS-UK [12, while the index $\xi$ distinguishes the left or right ion. Note that these coefficients, along with the coefficients making up the LCAO, are real.

When using the strong-field approximation, all structural information about the molecular orbital is contained in the recombination prefactor, $a_{\mathrm{rec}}(\mathbf{k}+\mathbf{A}(t))$, which is given by

$$
a_{\mathrm{rec}}(\mathbf{k})=\frac{1}{(2 \pi)^{3 / 2}} \int d^{3} r \mathbf{r} \cdot \hat{e}_{z} \exp [-i \mathbf{k} \cdot \mathbf{r}] \Psi(\mathbf{r}) .
$$

This is the component of $i \partial_{\mathbf{k}} \Psi(\mathbf{k})$ along the laser-field polarization. This prefactor gives rise to any two center interference which occurs in the harmonic spectra. The ionization prefactor, $a_{\text {ion }}\left(\mathbf{k}+\mathbf{A}\left(t^{\prime}\right)\right)$, on the other hand, will mainly determine whether tunnel ionization will be suppressed or enhanced due to the shape of a particular orbital. Therefore it directly affects the overall harmonic yield, but is not responsible for any interference patterns. Both prefactors are vanishing for nodes in the molecular orbitals.

In this work we use the momentum space wavefunctions to calculate the prefactors, which are given by

$$
\begin{aligned}
\psi(\mathbf{k}) & =\sum_{\alpha} \exp \left[i \mathbf{k} \cdot \frac{\mathbf{R}}{2}\right] c_{\alpha}^{(L)} \Phi_{\alpha}^{(L)}(\mathbf{k}) \\
& +(-1)^{l_{\alpha}-m_{\alpha}+\lambda_{\alpha}} \exp \left[-i \mathbf{k} \cdot \frac{\mathbf{R}}{2}\right] c_{\alpha}^{(R)} \Phi_{\alpha}^{(R)}(\mathbf{k})
\end{aligned}
$$

where

$$
\Phi_{\alpha}^{(\xi)}(\mathbf{k})=\sum_{\nu} b_{\nu}^{(\xi)} \tilde{\varphi}_{\nu}^{(\xi)}(\mathbf{k})
$$

with

$$
\tilde{\varphi}_{\nu}^{(\xi)}(\mathbf{k})=\left(-i k_{\beta}\right)^{l_{\alpha}} \frac{\pi^{3 / 2}}{2^{l_{\alpha}}\left(\zeta_{\nu}^{(\xi)}\right)^{3 / 2+l_{\alpha}}} \exp \left[-k^{2} /\left(4 \zeta_{\nu}^{(\xi)}\right)\right]
$$


Influence of asymmetry and nodal planes on high-harmonic generation in heteronuclear molecules5

Similarly to the position space expressions, $\beta=z, \beta=x$ and $\beta=y$ for the $\sigma$, $\pi_{x}$ and $\pi_{y}$ orbitals. The return condition (4) guarantees that the momentum $\mathbf{k}$ and the external field are collinear. Hence, for a linearly polarized field the angle between the intermediate momentum $\mathbf{k}$ and the internuclear axis $\mathbf{R}$ is equal to the alignment angle $\theta_{L}$ between the molecule and the field. The above-stated equations have been derived under the assumption that only $s$ and $p$ states will be employed in order to build the wavefunctions utilized in this work. For more general expressions see Ref. 13 .

\subsection{Interference Condition}

Maxima and minima in the momentum-space wavefunction may be determined by writing the exponents in Eq. (10) in terms of trigonometric functions. In this case, one obtains

$$
\psi(\mathbf{k})=\sum_{\alpha} \mathcal{C}_{+}^{(\alpha)} \cos \left[\frac{\mathbf{k} \cdot \mathbf{R}}{2}\right]+i \mathcal{C}_{-}^{(\alpha)} \sin \left[\frac{\mathbf{k} \cdot \mathbf{R}}{2}\right]
$$

with

$$
\mathcal{C}_{ \pm}^{(\alpha)}=(-1)^{l_{\alpha}-m_{\alpha}+\lambda_{\alpha}} c_{\alpha}^{(R)} \Phi_{\alpha}^{(R)}(\mathbf{k}) \pm c_{\alpha}^{(L)} \Phi_{\alpha}^{(L)}(\mathbf{k}) .
$$

Calling $\vartheta=\arctan \left[i \mathcal{C}_{+}^{(\alpha)} / \mathcal{C}_{-}^{(\alpha)}\right]$,we find

$$
\psi(\mathbf{k})=\sum_{\alpha} \sqrt{\left(\mathcal{C}_{+}^{(\alpha)}\right)^{2}-\left(\mathcal{C}_{-}^{(\alpha)}\right)^{2}} \sin [\vartheta+\mathbf{k} \cdot \mathbf{R} / 2] .
$$

Eq. (14) exhibit minima for $\vartheta+\mathbf{k} \cdot \mathbf{R} / 2=n \pi$.

Note, however, that the coefficients $\mathcal{C}_{ \pm}^{(\alpha)}$ defined in Eq. (13) depend on the wavefunctions at the left and right ions. Since these wavefunctions themselves depend on the momentum $\mathbf{k}$, one expects the two-center patterns to be blurred for heteronuclear molecules. In contrast, for homonuclear molecules, $c_{\alpha}^{(L)}=c_{\alpha}^{(R)}$ and

$\Phi_{\alpha}^{(L)}(\mathbf{k})=\Phi_{\alpha}^{(R)}(\mathbf{k})$. This implies that the momentum dependence in the argument $\vartheta$ cancels out and that the interference condition in Refs. 8, 13] is recovered. In this case, sharp interference fringes are expected to be present.

The above-stated condition does not only lead to interference fringes in the boundstate momentum wavefunctions but also in the high-harmonic spectra. This is due to the fact that the dipole matrix elements depend on the wavefunctions (10). In this latter case, $\mathbf{k} \rightarrow \mathbf{k}+\mathbf{A}(t)$ in Eqs. (10)-(14).

For a linearly polarized monochromatic field of frequency $\omega$, using the saddlepoint equation (5), the generalized interference condition (14) may be expressed in terms of the harmonic order $n$ as

$$
n=\frac{E_{0}}{\omega}+\frac{2(\kappa \pi-\vartheta)^{2}}{\omega R^{2} \cos ^{2} \theta_{L}},
$$

where $E_{0}$ is the absolute value of the bound-state energy in question, $\kappa$ is an integer number, $\theta_{L}$ is the alignment angle, $R$ is the internuclear distance and $\vartheta$ is defined above). This interference condition has been first derived in [18 and has been used by us in previous work [13].

In the specific case of orbitals of $\pi_{g}$ type, which are constructed entirely from ptype atomic orbitals such that $l_{\alpha}=1$, are of gerade symmetry such that $\lambda_{\alpha}=0$, and are made from a homonuclear species such that $\Phi_{\alpha}^{(R)}(\mathbf{k})=\Phi_{\alpha}^{(L)}(\mathbf{k})$ and $c_{\alpha}^{(R)}=c_{\alpha}^{(L)}$, 

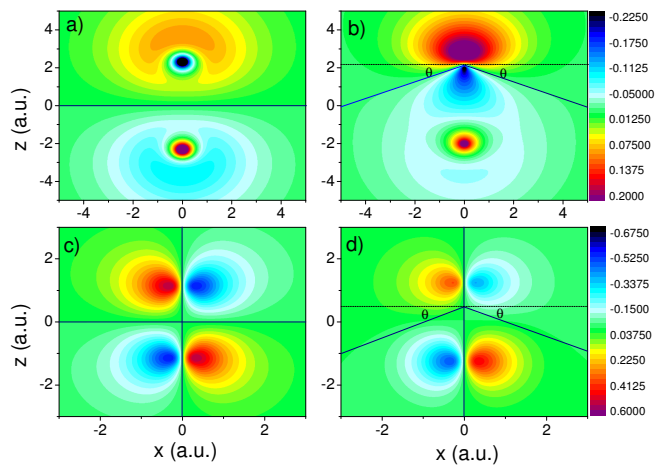

Figure 1. Highest occupied molecular orbitals in position space for the homonuclear molecules $\mathrm{Be}_{2}$ and $\mathrm{O}_{2}$ [panels a) and c)], and their heteronuclear isoelectronic counterparts $\mathrm{LiB}$ and NF [panels b) and d)]. The orbital symmetries in panels a), b), c) and d) are $2 \sigma_{u}, 4 \sigma, 1 \pi_{g}$ and $2 \pi$, respectively, and the bond lengths are $R^{\left(\mathrm{Be}_{2}\right)}=4.642$ a.u., $R^{(\mathrm{LiB})}=4.642$ a.u., $R^{\left(\mathrm{O}_{2}\right)}=2.280$ a.u., and $R^{(\mathrm{NF})}=2.485$ a.u.

one finds that $\vartheta=0$. This implies that for the zeroth order interference, where $\kappa=0$, the second term of (15) will vanish leaving the angular independent term

$$
n=\frac{E_{0}}{\omega} \text {. }
$$

This minimum, however, would occur exactly at the ionization threshold, for which the present framework is expected to break down. Hence, it will not be discussed here.

\section{Results}

\subsection{Position and momentum space wavefunctions}

In this section, we will display the specific molecular orbitals employed by us in the results that follow. We choose two pairs of molecules composed of a homonuclear molecule and an isoelectronic heteronuclear molecule. The first pair, $\mathrm{Be}_{2}$ and $\mathrm{LiB}$, possess eight electrons and a $\sigma$ highest occupied molecular orbital, while the second pair, $\mathrm{O}_{2}$ and NF, have sixteen electrons and a $\pi$ highest occupied molecular orbital.

In Fig. 11, we exhibit the HOMO for homonuclear molecules, $\mathrm{Be}_{2}$ and $\mathrm{O}_{2}$, and heteronuclear molecules, LiB and NF, as obtained with GAMESS-UK [Figs. 1. (a),(b),(c) and (d) respectively]. The internuclear axis is aligned along the $z$-axis. The position-space wavefunctions of $\mathrm{Be}_{2}$ and $\mathrm{LiB}$ are of $\sigma$ type with $\mathrm{Be}_{2}$ having ungerade symmetry, $\sigma_{u}$, and a nodal plane along the $x$-axis at $z=0$. For LiB, there is a bias towards the Boron atom. One can also clearly see the contribution of the $\mathrm{p}$ type atomic orbital which is introduced by the Boron atom. The position-space wavefunctions of $\mathrm{O}_{2}$ and NF are of $\pi$ type with $\mathrm{O}_{2}$ having gerade symmetry, $\pi_{g}$, and a nodal plane along the $\mathrm{x}$-axis at $z=0$ and the z-axis at $x=0$. NF shows a bias towards the Fluorine atom.

We have found that both momentum-space wavefunctions, given in Figs. 2$] 3$ and 4 are symmetric with respect to $\left(p_{x}, p_{z}\right) \rightarrow\left(-p_{x},-p_{z}\right)$ in comparison to their 

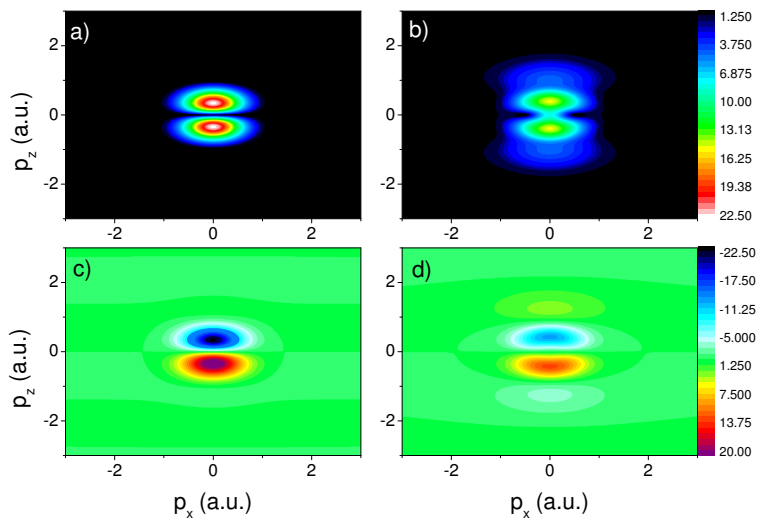

Figure 2. Absolute values and imaginary parts of the highest occupied molecular orbitals in momentum space for the homonuclear molecule $\mathrm{Be}_{2}$ [panels a) and c) respectively] and its heteronuclear counterpart $\mathrm{LiB}$ [panels b) and d)].

position-space counterparts. Despite that, the momentum-space wavefunctions for homonuclear and heteronuclear molecules exhibit different properties, especially if a real basis set is used in the construction of the position-space wavefunctions $\psi(\mathbf{r})$. They come from the properties of Fourier transforms and will determine whether a particular node will be blurred in the heteronuclear case. An even wavefunction in position space will lead to a real wavefunction in momentum space, as the Fourier transform of a real and even function should be real. For the same reason, if the positionspace wavefunction is odd, the corresponding momentum-space wavefunction should be pure imaginary. Neither property will hold if the position-space wavefunction does not possess a well-defined symmetry. In this case, its momentum-space counterpart will be complex.

We will now have a closer look at the nodes in the momentum-space wavefunctions. For $\mathrm{Be}_{2}$, the momentum-space wavefunction of the $\sigma_{u} \mathrm{HOMO}$ is pure imaginary, and exhibit a clear node along the $p_{x}$-axis (see Figs. 2. (a) and (c) for $|\psi(\mathbf{k})|$ and $\operatorname{Im}[\psi(\mathbf{k}]$, respectively). This node is caused by the linear combination of atomic orbitals giving rise to the $\sigma_{u}$ orbital.

The above-mentioned node is lost in the absolute value of the HOMO wavefunction of $\mathrm{LiB}$, shown in Fig. 2 (b), even though a suppression along the $p_{x}$ axis is still present. Apart from that, there is a secondary set of peaks extending towards higher momentum values. One should note, however, that a very clear central node for $\mathrm{LiB}$ at the $p_{x}$ axis can be seen in the imaginary part of corresponding wavefunction, shown in Fig. 2(d). This feature is blurred by the real part of $\psi(\mathbf{k})$, which does not have this node, and is of comparable magnitude (see Fig. 团(a)). As discussed above, a complex wavefunction in momentum space is related to an asymmetric wavefunction in position space. Hence, the loss of symmetry in position space is what leads to the loss of the clear nodal plane in momentum space. Physically, the asymmetry in the position-space wavefunction can be traced back to the static dipole moment along the internuclear axis. 

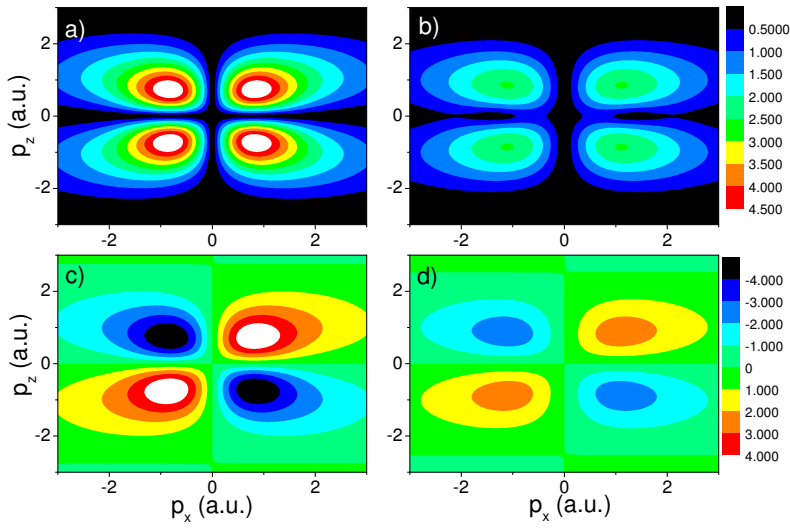

Figure 3. Absolute values and real parts of the highest occupied molecular orbitals in momentum space for homonuclear molecule $\mathrm{O}_{2}$ [panels a) and c) respectively] and its heteronuclear counterpart NF [panels b) and d)].
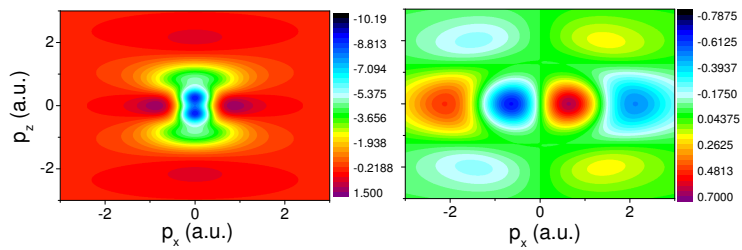

Figure 4. Real part of the highest occupied molecular orbital in momentum space for LiB and imaginary part of the highest occupied molecular orbital in momentum space for NF, panels a) and b) respectively.

In comparing $\mathrm{O}_{2}$ and NF momentum space wavefunctions as shown in Fig. 3, one can see that the features are quite similar for one of the nodal planes. Specifically, the well defined nodal plane along the $p_{x}$-axis observed for $\mathrm{O}_{2}$ has been slightly blurred for NF (see Figs. 3. (a) and (b), respectively). This again is because of the loss of symmetry in the heteronuclear case, due to the static dipole moment. Note once more that an even and real position-space wavefunction, such as the HOMO in $\mathrm{O}_{2}$, leads to a real momentum-space wavefunction. This is not the case for NF, and the non-vanishing imaginary part of the momentum space wavefunction gives rise to

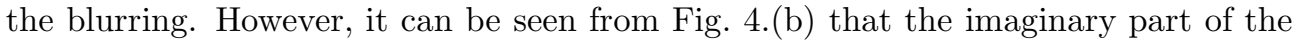
momentum space wavefunction for NF is much smaller than its real part. This implies that the NF molecule is quite close to having gerade type symmetry, and therefore one would expect similar features in the position and momentum space wavefunctions as compared to $\mathrm{O}_{2}$.

In contrast, the other nodal plane, along the $z$-axis, remains completely unaffected 
Influence of asymmetry and nodal planes on high-harmonic generation in heteronuclear molecules 9

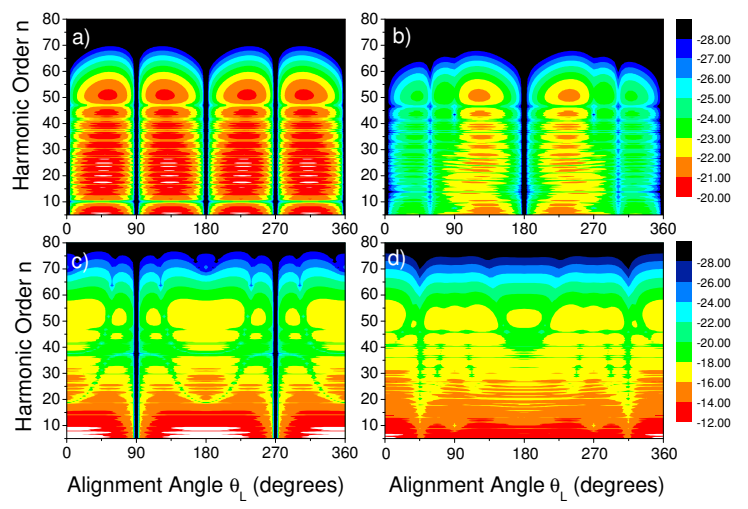

Figure 5. Harmonic spectra as a function of alignment angle for the molecule a) $\mathrm{Be}_{2}$ of ionization potential 0.2390 a.u., b) $\mathrm{LiB}$ of ionization potential 0.1942 a.u., c) $\mathrm{O}_{2}$ of ionization potential 0.2446 a.u. and d) NF of ionization potential 0.2246 a.u. A linearly polarized laser field of frequency $\omega=0.057$ a.u. and intensity $I=4 \times 10^{14} \mathrm{~W} / \mathrm{cm}^{2}$ is used. The spectra have been computed using the length gauge and employing the dipole operator in the length form.

by the presence of the static dipole moment. Indeed, this nodal plane is just as pronounced in both molecules, irrespective of being homonuclear or heteronuclear. Mathematically, this is because one can define two types of nodal planes in position space: one of which is due to the characteristic of the $p$-type atomic orbitals, which are of the form $\sum_{\nu} b_{\nu}^{(\xi)} x e^{-\zeta_{\nu}^{(\xi)} r^{2}}$, and therefore disappear at $x=0$, irrespective of the contraction coefficients and exponential coefficients, and the other of which is due to the linear combination of atomic orbitals. The latter results in a cancelation for a homonuclear molecule, and hence a nodal plane. This however does not occur for a heteronuclear molecule. The nodal plane along the $p_{z}$-axis is of the first type, and is therefore present for both molecules, and the nodal plane along the $p_{x}$-axis is of the second type and not present for NF. This argument can be mapped into the momentum space using Fourier transforms (see Eq. (10)).

\subsection{Harmonic Spectra}

We now present the harmonic spectra computed in a linearly polarized monochromatic wave of frequency $\omega$ and amplitude $\omega A_{0}$, for all molecules discussed previously. These results are displayed in Fig. 5. It can be seen that all spectra contains a series of cuts, i.e., a complete suppression of the yield for particular angles. Most notably in the case of $\mathrm{O}_{2}$ these occur at alignment angles $\theta_{L}=0,90,180,270$ and 360 degrees (see Fig. 5. (a)). By observing the position space wavefunction in Fig. 1. (c) and the absolute value of the momentum space wavefunction in Fig. 3. (a) this can immediately 
be attributed to the nodal planes of the molecular wavefunction, which occur at these angles, and therefore prohibit ionization or recombination. The situation in the case of NF, however, is modified, as shown in Fig. 5. (b). The harmonic signal completely vanishes at alignment angles $\theta_{L}=0,180$ and 360 degrees, because at these angles the nodal planes in the highest occupied molecular orbital occur due to the nature of the $p$-type atomic orbitals. However, due to the polar nature of these heteronuclear molecules, and a linear combination of atomic orbitals which no longer cancels out, the nodal planes at alignment angles $\theta_{L}=90$ and 270 degrees are no longer present.

In fact, several differences with regard to the homonuclear case are observed. Firstly, the signal is no longer completely suppressed, but there is a distinct minimum which occurs at alignment angles $\theta_{L}=54$ and 306 degrees. There is also a second minimum, but not a cut, beyond harmonic $\mathrm{n}=35$, which occurs at the alignment angles $\theta_{L}=90$ and 270 degrees, that is, where the nodes were in the homonuclear molecule. We attribute this to the remnants of the nodal plane in its heteronuclear counterpart. This can be seen more clearly in Fig. (7)(a), where the harmonic spectra of $\mathrm{O}_{2}$ and NF are plotted as functions of the alignment angle for fixed harmonic order.

Inspection of the position and momentum space wavefunction suggests that the polar nature of the heteronuclear molecules deforms the nodal plane such that, although there is no longer a node, there is a suppression which occurs at the angle to which the plane has been deformed. As the molecule is rotated the new minimum in the wavefunction is first experienced before $\theta_{L}=90$ degrees but then occurs at the same angle after $\theta_{L}=270$ degrees. Therefore the spectra has reflectional symmetry about an alignment angle of 180 degrees. There is no longer a clear nodal plane, as the probability density associated to the wavefunction is small, but nonvanishing. Hence, there is not a complete suppression in the harmonic spectra. However, a clear minimum is still visible.

Note that, for $\mathrm{O}_{2}$, the two-center interference maxima and minima predicted by Eq. (16) are located either beyond the cutoff or at the ionization threshold. Therefore, they do not occur in the parameter range of interest. This also holds for its heteronuclear counterpart.

Now observing the spectra for $\mathrm{Be}_{2}$, displayed in Fig. [5.(c), one may identify nodes at $\theta_{L}=90$ and 270 degrees, as one would expect by observing the position and momentum space wavefunctions. In the spectra of the heteronuclear counterpart LiB, presented in Fig. 5. (d), these nodes have been replaced by minima at $\theta_{L}=45$ and 315 degrees, for the same reasons as described above.

The spectra for $\mathrm{Be}_{2}$ also exhibit two-center interference minima, corresponding to $\kappa=0, \kappa=1$ and $\kappa=2$ in Eq. (15), whose energy position depends strongly on the alignment angle. These are shifted considerably in the heteronuclear counterpart $\mathrm{LiB}$. This is because the $\mathrm{Be}_{2}$ molecular wavefunction is constructed almost entirely of $s$-type atomic orbitals, whereas in the case of LiB the Boron atom introduces $p$-type atomic orbitals into the molecular wavefunction and hence changes the interference condition. Note that, with regard to $\mathrm{Be}_{2}$, there is a blurring in the two-center patterns for LiB. This is expected according to the discussion in Sec. 2.3.

This is shown in more detail in Fig. [6, in which the individual contributions of the $s$ and $p$ states to the spectra of $\mathrm{Be}_{2}$ and $\mathrm{LiB}$ are presented (upper and lower panels, respectively). For these individual contributions, there is also a considerably simpler interference condition for homonuclear molecules, which can be obtained from Eq. (15) by setting either $C_{+}$or $C_{-}$equal to zero. Specifically the $s$-state and $p$-state 


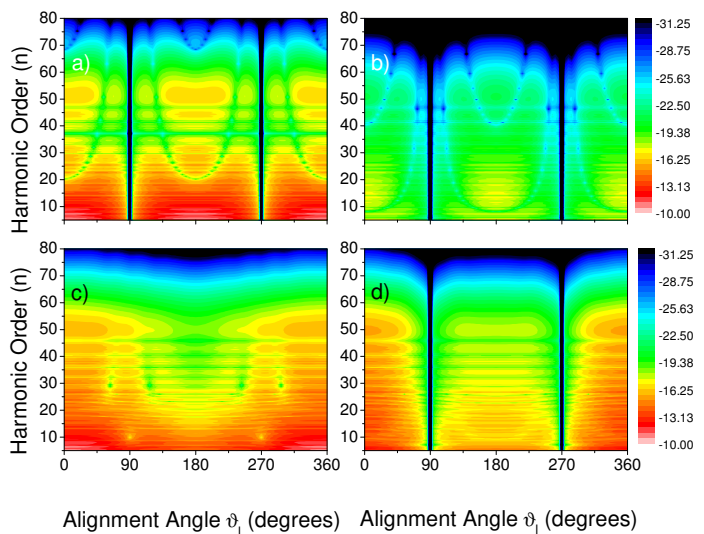

Figure 6. Harmonic spectra as a function of alignment angle for a) the s-type orbitals of the $\mathrm{Be}_{2} \mathrm{HOMO}, \mathrm{b}$ ) the p-type orbitals of the $\mathrm{Be}_{2} \mathrm{HOMO}, \mathrm{c}$ ) the s-type orbitals of the LiB HOMO, d) the p-type orbitals of the LiB HOMO, for the same parameters as in the previous figure.

contributions for an ungerade orbital exhibit minima at the harmonic frequencies

$$
\Omega_{s}^{(u)}=E_{0}+\frac{2 \kappa^{2} \pi^{2}}{R^{2} \cos ^{2} \theta_{L}}
$$

and

$$
\Omega_{p}^{(u)}=E_{0}+\frac{(2 \kappa+1)^{2} \pi^{2}}{2 R^{2} \cos ^{2} \theta_{L}}
$$

respectively, where $\kappa$ is a integer. In Fig. 6)(a), we may identify the minima for $\kappa=1$ and $\kappa=2$ according to Eq. (17), while in Fig. 6) (b) the minima corresponding to $\kappa=0$ and $\kappa=1$ can be easily found. If the s p mixing is considered, the full expression (15) must be used.

The contributions from $p$-type orbitals to the harmonic spectrum of $\mathrm{Be}_{2}$ are orders of magnitude smaller than the contribution of p-type orbitals in LiB. Therefore the overall spectrum of $\mathrm{Be}_{2}$ is dominated mostly by the s-type atomic orbitals. In contrast, in $\mathrm{LiB}$, both types of orbitals lead to comparable contributions to the spectra, and the $s p$ mixing will influence the energy position of the overall two-center patterns. Apart from that, the ionization potentials and internuclear separation of both molecules are slightly different. This will influence the two-center interference further.

The effects of $s p$ mixing are also displayed in Fig. 7.(b) and Fig. 7.(c) for a fixed mid-plateau harmonic. The figure clearly shows that where one observes that, for $\mathrm{Be}_{2}$, the $s$-type orbitals dominate and the main effect of the $p$-type orbitals is to introduce a small shift in the interference minima. On the other hand, for $\mathrm{LiB}$, the overall maxima and minima are considerably altered by $s p$ mixing. In the figure, one can also see that the nodal planes occurring at alignment angles $\theta_{L}=90$ and 270 degrees in $\mathrm{Be}_{2}$, are shifted by the contribution of the s-type orbitals in $\mathrm{LiB}$.

Finally, we will comment on the overall harmonic intensities. The harmonics generated from $\mathrm{NF}$ are less intense than those from $\mathrm{O}_{2}$. As the ionization potential of 


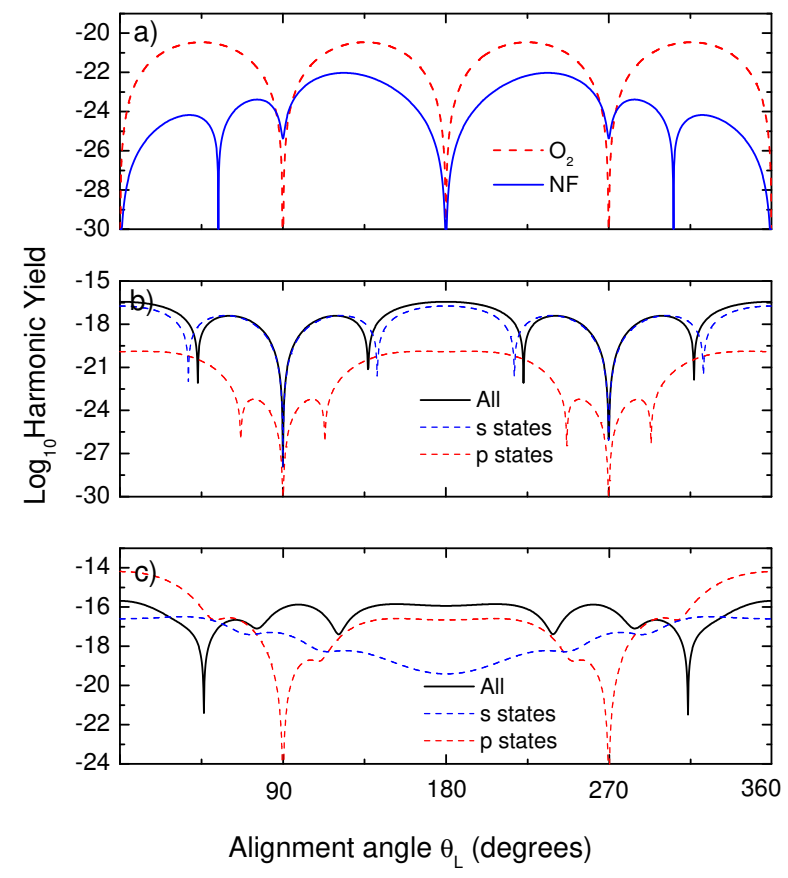

Figure 7. The harmonic yield of a) $\mathrm{O}_{2}$ and $\mathrm{NF}$ for the $45^{\text {th }}$ harmonic vs the alignment angle, b) $\mathrm{s}$ and $\mathrm{p}$ orbitals to the harmonic yield of $\mathrm{Be}_{2}$ for the $25^{\text {th }}$ harmonic vs the alignment angle and c) s and p orbitals to the harmonic yield of $\mathrm{LiB}$ for the $25^{\text {th }}$ harmonic vs the alignment angle. All parameters are the same as in the previous figure.

$\mathrm{NF}$ is slightly lower than that of $\mathrm{O}_{2}$, one would expect more ionization and therefore higher harmonic yield. This implies that the shape of the molecular wavefunction results in suppression at the recombination step for $\mathrm{NF}$, an effect which is more prominent than the difference in ionization potential. Harmonics generated from $\mathrm{Be}_{2}$ and $\mathrm{LiB}$ are much more intense than those generated by $\mathrm{O}_{2}$ and $\mathrm{NF}$, despite similar ionization potentials. This suggests that the presence of nodal planes, or minima associated with strong suppression of the wave function, reduce the yield of the entire spectrum. As $\mathrm{O}_{2}$ has two nodal planes and $\mathrm{Be}_{2}$ has one, harmonic spectra from the former is less intense than that of the latter.

\section{Conclusions}

In this work, we investigated the dependence of the high-order harmonic spectra on the alignment angle between the diatomic molecules and the laser-field polarization, for isoelectronic pairs consisting of a homonuclear and a heteronuclear molecule. We employed a single active electron approximation, using the HOMO as the active orbital, within the strong-field approximation.

Our studies lead us to conclude that there are two types of nodes in the bound- 
state orbitals of molecules, which will lead to a strong suppression in the harmonic yield when such nodes are parallel to the laser-field polarization. The first type is caused by the nodes in the atomic orbitals at each ion, and is present at the same location for homonuclear molecules and their heteronuclear counterparts. The second type of node is due to the sum or subtraction of atomic orbitals at different centers within the linear combination of atomic orbital (LCAO) approximation. Both types of minima are present for homonuclear molecules. For their heteronuclear counterparts, however, the asymmetry of the molecule eliminates the latter nodes. This implies that the imprints of the first type of nodes in the harmonic spectra are common to isoelectronic homonuclear and heteronuclear molecules, and could in principle be observed in both cases.

In general, the asymmetry also leads to some blurring in the interference patterns caused by high-harmonic emission at spatially separated centers in the molecule. Furthermore, depending on the molecule, $s p$ mixing will be different. This will lead to shifts in the energy positions of the two-center patterns. On a more technical level, we have also been able to map the symmetry or asymmetry of the molecular orbitals in position space to properties of their momentum-space counterparts.

The above-stated findings show that nodal planes in a heteronuclear molecule can be related to those in an isoelectronic homonuclear molecule. Hence, in principle, the latter can be used as a reference point in order to understand the behavior of the former, by using high-order harmonic generation. The shifts in the nodal planes due to the distortions in the wavefunctions or the absence thereof can be mapped into features in the high-harmonic spectra. This may shed some light in the imaging of heteronuclear molecules.

\section{Acknowledgments}

We thank H. J. J. van Dam, P. J. Durham, P. Sherwood and J. Tennyson for very useful discussions. This work was supported by the UK Engineering and Physical Sciences Research Council (EPSRC), and by the Daresbury Laboratory.

[1] McPherson A, Gibson G, Jara H, Johann U, Luk T S, McIntyre I A, Boyer K, and Rhodes C K 1987 J. Opt. Soc. Am. B 4, 595.

[2] Lewenstein M, Balcou Ph, Ivanov M Yu, L'Huillier A and Corkum P B 1994 Phys. Rev. A 49, 2117.

[3] Corkum P B 1993 Phys. Rev. Lett. 71, 1994.

[4] Kanai T, Minemoto S and Sakai H 2005 Nature 435, 470.

[5] Itatani J, Levesque J, Zeidler D, Niikura H, Pépin H, Kieffer J C, Corkum P B and Villeneuve D M 2004 Nature 432, 867.

[6] Smirnova O, Mairesse Y, Patchkovskii S, Dudovich N, Villeneuve D, Corkum P B, Ivanov M Y 2009 Nature 460, 972.

[7] Lein M, Hay N, Velotta R, Marangos J P, and Knight P L 2002 Phys. Rev. Lett. 88, 183903; 2002 Phys. Rev. A 66, 023805; Spanner M, Smirnova O, Corkum P B and Ivanov M Y 2004 J. Phys. B 37, L243.

[8] Milošević D B 2006 Phys. Rev. A 74, 063404; Busuladžić M, Gazibegović-BusuladžićA, Milošević D B, and Becker W 2008 Phys. Rev. Lett. 100, 203003; 2008 Phys. Rev. A 78, 033412.

[9] Madsen C B and Madsen L B, 2006 Phys. Rev. A 74, 023403; Madsen C B, Mouritzen A S, Kjeldsen T K, and Madsen L B, 2007 Phys. Rev. A 76, 035401.

[10] Chu X and Chu S I, 2001 Phys. Rev. A 64, 063404; Telnov D A and Chu S- I 2009, Phys. Rev. A 80, 043412; Chu X and Chu S I; 2004 Phys. Rev. A 70, 061402; Son S -K and Chu S I 2009 Phys. Rev. A 80, 011403.

[11] Holmegaard L, Hansen J L, Kalhj L, Kragh S L, Stapelfeldt H, Filsinger F, Kpper J, Meijer G, Dimitrovski D, Abu-samha M, Martiny C P J, and Madsen L B 2010 Nature Phys. 6, 428; Etches A and Madsen L B 2010 J. Phys. B 43, 155602. 
Influence of asymmetry and nodal planes on high-harmonic generation in heteronuclear molecules14

[12] GAMESS-UK is a package of ab initio programs. See: "http://www.cfs.dl.ac.uk/gamessuk/index.shtml", Guest M F, Bush I J, van Dam H J J, Sherwood P, Thomas J H, van Lenthe J H, Havenith R W A, Kendrick J 2005 Mol. Phys. 103, 719.

[13] Figueira de Morisson Faria C and Augstein B B 2010 Phys. Rev. A 81, 043409.

[14] Patchkovskii S, Zhao Z, Brabec T and Villeneuve D M 2006 Phys. Rev. Lett. 97, 123003; Patchkovskii S, Zhao Z, Brabec T, and Villeneuve D M, 2007 J. Chem. Phys. 126, 114306; Smirnova O, Patchkovskii S, Mairesse Y, Dudovich N, Villeneuve D, Corkum P B, and Ivanov M Yu 2009 Phys. Rev. Lett. 102, 063601.

[15] Figueira de Morisson Faria C 2007 Phys. Rev. A 76, 043407; Smirnova O, Spanner M and Ivanov M, 2007 J. Mod. Opt. 54, 1019.

[16] Figueira de Morisson Faria C, Schomerus H and Becker W 2002 Phys. Rev. A 66, 043413.

[17] Augstein B B and Figueira de Morisson Faria C, arXiv: 1007.2135 [atom.ph]

[18] Odžak S and Milošević D B 2009 Phys. Rev. A 79, 023414; 2009 J. Phys. B 42, 071001. 